\title{
Interactive comment on "Glacier change and glacial lake outburst flood risk in the Bolivian Andes" by Simon J. Cook et al.
}

\section{Simon J. Cook et al.}

s.j.cook@mmu.ac.uk

Received and published: 15 September 2016

Reply to reviewer 2

We thank the reviewer for their thoughtful comments on our manuscript. We are pleased that they suggest our work be published, and that they praise the approach and merit of the work. We address the specific and minor comments below.

Specific comments

We agree that care should be taken when reporting statistics about water resources and vulnerability. It is easy to regurgitate these figures and lose a sense of the science upon which they are based, and the reviewer is right to check that we are using language and statistics appropriately. The reviewer first points to a specific example 
on p1-28 of the original manuscript. We should clarify that the values cited here are based on previous work, but we had failed to cite the accompanying reference (Soruco et al., 2015), which made the value look arbitrary. We have now added that reference (p1-28). We have also added further discussion of the results of Soruco et al (2015) into section 4.1 because they studied decadal changes (or lack of) in runoff to La Paz (P10 L2-5). We have added additional references (e.g. Painter, 2007) to the Introduction to back-up points that we had made (P1-29). I would argue that, overall, we have been careful and conservative in our writing - more so than in previous studies in some cases. For example, the reviewer suggests that some values are speculative without measured values, but we already state that further quantification is required (e.g. P22). We are also quite careful not to be alarmist - we use phrases like "La Paz... derives *some* of its electricity from hydropower generation, which depends to *some

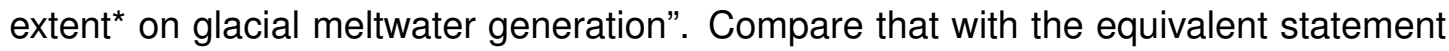
in Painter (2007): "La Paz is also dependent for *virtually all* of its energy supplies on hydroelectric power". One of us (Hoffmann) lives in La Paz, and knows that this latter statement is incorrect, and hence we toned-down our writing of the importance of glaciers for hydropower in Bolivia. Elsewhere, we have toned-down our language. For example, in the abstract and introduction we had said that meltwater was "vital", but now we say it is "important"( $\mathrm{P} 1-1$ \& 25). We hope that the addition of some missing references, along with further discussion of the few papers that have quantified glacier and runoff change (e.g. Soruco et al., 2015) will satisfy the reviewer here.

The reviewer comments on our process for identifying potentially dangerous lakes. We think that it is a good idea to include a flow diagram that summarises the process of identifying such lakes. We had considered this before submitting our manuscript, but decided against it on the basis of article length and page costs. We have, however, adopted this suggestion - see Figure 2. The reviewer's suggestion that we consider ranking the lakes in some way according to their level of danger is a good one, and this is something that we intend to work on as part of the second author's PhD project. However, there are, as yet, no universally accepted or adopted methods for determining

Printer-friendly version

Discussion paper 
how dangerous a lake is - should population exposure be a more important factor than lake size? Should the potential for rockfall into a lake be of greater concern than a degrading ice-cored moraine? Because this is such a complex issue, and because we simply wanted to identify lakes that are potentially dangerous (rather than measure their absolute level of danger/threat), we opted for a simpler approach - potential flood size - as the reviewer correctly identified. We have clarified in the text that we use peak discharge to order the lakes.

The reviewer comments on our error estimates. We adopted the technique outlined in Hanshaw and Bookhagen (2014), which calculates error as a function of glacier size (perimeter) and spatial resolution of the imagery. Hence, a relatively small total perimeter could yield a larger error estimate. For the most part, our glacier mapping was undertaken automatically. As reported in previous studies, automatic mapping yields errors typically between 2 to 6\% (Paul et al., 2013). But again, we have been conservative here in our error estimate because we corrected some mapping manually. In reality, we expect the error to be lower than 10\%. Hence, we cited Paul et al. (2013) with their error estimates for clean glaciers of $\sim 5 \%$.

We are glad the reviewer likes the kmz file - we hope this will prove to be a useful resource for anyone interested in the issues raised in our manuscript.

The reviewer suggests we look into reasons why patterns of lake development over time are not straightforward. Certainly, this is something that we are interested in, and intend to follow-up on in our continuing work. But we are keen to keep this manuscript focused on the three stated objectives outlined in the Introduction. The second objective was to evaluate lake development (rather than explain it fully), which we have achieved. This is principally to allow us to move on to objective 3 to identify potentially dangerous lakes. Some interesting patterns emerge from our evaluation of lake development, but to explain these patterns could become a study in its own right. We are grateful for the suggestions and enthusiasm of the reviewer on how such a study could be approached. We will follow-up on this.

Printer-friendly version

Discussion paper
Interactive comment 
The reviewer asks us to clarify whether we consider moraine-dammed lakes to be more dangerous than bedrock-dammed lakes. As we state in section 2.3 (P5 L31), moraine-dammed lakes are generally considered to be more dangerous because there is the potential for breach incision through the moraine thickness, allowing for a greater volume of water to escape from the lake. But we also make the point that bedrockdammed lakes can be sources of GLOFs, and cite some examples of this. So both are potentially dangerous, although there is a general consensus (as far as we are aware) that moraine-dammed lakes represent the greater threat. Hence, we categorised lakes by dam type in Table 2.

The reviewer questions our ordering or lakes in Table 2. Again, we have now clarified in the text that we have simply ranked the lakes by peak discharge (P6 L25). This is because there are no universally accepted methods of ranking lakes by threat - this is something we are working on, however.

The reviewer asks us to reconsider our use of the word "trend" in relation to ice-contact lake data, which show no strong tendency. This is a fair point, and we have adjusted our language accordingly (e.g. by using the word "change" instead). E.g. P11 L9.

Minor comments

P3 L5: We have now changed this to "initial assessment". P3 L6 \& P4 L23.

P4L26: We have added a colon. P4L28

P5 L23: We weren't sure what was being commented on here, so we have kept this the same.

P5 L27: We had to change this anyway in response to another comment - it now reads "lakes confined within". P5 L34.

P8 L21: Changed as requested. P8 L29

P10 L27: Changed as requested. P11 L9 
P11 L28: Changed as requested. P12 L12

P11, L32,33: Yes. Done. P12 L16-18

Map figures: We experimented with different map formats in preparing this manuscript - different ESRI backgrounds, Landsat imagery - natural and false colour. One of the issues is scale: our area of interest is very large, but the glaciers and lakes relatively small. If we were to plot up all of the locations of settlements from geobolivia for this region, it would somewhat dominate the map (essentially, a lot of dots). This is one of the reasons why we produced the supplementary $\mathrm{kmz}$ file so that people could look at the detail for themselves, whilst leaving the map figures fairly simple in order to illustrate glacier change and lake locations.

Table 2: Please see our response to earlier comments. We prefer to keep this very simple and order by peak discharge. Thanks for the space-saving tip. We saved a lot of space anyway in dealing with Reviewer 1's comments to remove the values derived from lake volume-area self-correlation. But we also changed the heading to "Dam type" to allow us to shorten the classifications to "moraine" and "bedrock".

Interactive comment on The Cryosphere Discuss., doi:10.5194/tc-2016-140, 2016. 